\title{
I2. COMMISSION DE LA RADIATION SOLAIRE ET DE LA SPECTROSCOPIE SOLAIRE
}

Préstdent: M. G. Righini.

Membres: MM. Abbot, Abetti, H. D. Babcock, Barabashev, Mlle Barbara Bell, MM. Brück, P. Carrasco, Chalonge, Chapman, d'Azambuja, Edlén, J. W. Evans, Evershed, Gnevyshev, Goldberg, Greenstein, Houtgast, Locke, Melnikov, Menzel, Migeotte, Minnaert, Mustel, Nicholson, Nicolet, A. K. Pierce, Plaskett, Richardson, Ruttlant, Schwarzschild, Severny, Sitnik, Suemoto, ten Bruggencate, Thiessen, von Klüber, Waldmeier, Wildt, Woolley.

This Report summarizes the research which has been made on solar radiation and solar spectroscopy from 1952 to the end of 1954 as derived from publications and from personal letters of the members of the commission. Owing to the similarity of the subjects treated by this commission with those treated by Commissions Io, II and $\mathrm{r}_{3}$, some duplication is unavoidable.

\section{GENERAL}

We want to draw attention to the following books which should be of particular interest to research workers in the field of solar radiation and solar spectroscopy:

The Sun, edited by G. P. Kuiper;

Rocket Exploration of the Upper Atmosphere, edited by R. L. Boyd and M. J. Seaton.

\section{Energy Wave-Length Curve}

The ultra-violet part of the spectrum has been investigated by means of spectrographs carried in the high atmosphere by rockets. Johnson, Purcell, Tousey and Wilson obtained an absolute spectral curve between 2000 and $3200 \AA$, comparing solar spectra with a carbon arc, the spectral distribution of which is known from laboratory research. From 3200 to $6000 \AA$ new measurements with a quartz monochromator and photomultiplier have been secured by Scolnik and Dunkel at Mount Lemmon (Arizona). Reduction to zero air mass has been applied.

Stair, Johnston and Blagg measured a spectral curve of the Sun in the range from 2990 to $5350 \AA$ at Sacramento Peak (New Mexico), comparing the Sun with a tungsten ribbon lamp with quartz envelope. In the region from 5000 to 25,000 $\AA$ explored with a $\mathrm{PbS}$ cell, the results are only provisional.

At Mount Wilson Pierce measured the radiation of the centre of the disk employing the McMath-Hulbert infra-red spectrograph attached to the Snow telescope. The measurements have been made on the true continuum between the lines.

It appears that the Sun radiates more energy at $16,000 \AA$ than at 10,000 or $25,000 \AA$ as compared with a black body. The increased emission at $16,000 \AA$ is in agreement with expectation if the $\mathrm{H}^{-}$is the principal source of opacity.

Absolute measurements in the solar spectrum by comparison with a black body in vacuo are in progress at the Institut d'Astrophysique in Paris by Peytureaux. Photoelectric researches on the continuous spectrum have been carried out since 1952 at the Kutchino Observatory (U.S.S.R.).

\section{Solar Constant}

Aldrich and Hoover, continuing the work of Abbot, have published new measurements of the solar constant. A period of thirty years of solar constant values is now complete. During the last six years the pyrheliometer constants have been revised with the result that the actual values of the solar constant are only $0.7 \%$ too high. The mean solar constant deduced from the records of thirty years is 1.932 Langleys/min. A revision of the U.V. and I.R. corrections, as suggested by many authors, leads to an increase of $0 \cdot 4 \%$. 
A solar constant of $2 \cdot 00 \pm 0 \cdot 04$ Langleys/min. has been obtained by Johnson and collaborators from the integration of a spectral curve. Johnston, Stair and Blagg, discussing their observations, arrived at the same result.

According to Aldrich and Hoover there is an increase in the solar constant with increasing sunspot number. According to Abbot the weather is seriously affected by the general radiation of the Sun and the solar variation has a master period of about twentytwo years.

Discussing the pyrheliometric measurements made at Mount Stromlo (Australia) during the period 1927-39, Rimmer and Allen reach the conclusion that 'the solar variations are not great enough to be detectable by pyrheliometric measurements'.

\section{Centre-limb Darkening}

New measurements have been performed by:

Peytureaux for twenty-five wave-lengths between 3 I90 and 23,r30 $\AA$ with measurements in eighteen points of the radius up to $\sin \theta=0.985$.

Ramanathan in the visible spectrum. Photographic method, measurements up to $\sin \theta=0.98$.

Pierce at the Snow telescope (Mount Wilson) with the McMath spectrometer and $\mathrm{PbS}$ cell. Measurements in twenty-six wave-lengths covering the interval from 7793 to $24,388 \AA$ for points ranging from $\cos \theta=\mathrm{I}$ to $\cos \theta=0 \cdot$ ro. Other measurements in the spectral range $38 \mathrm{II}-6000 \AA$.

Jäger in the visible. Photographic method of Unsöld-ten Bruggencate at the partial eclipse of 30 June I954. Up to the extreme limb.

Ballario and Godoli at the partial eclipses I September I95I and 30 June I954. Unsöld-ten Bruggencate method, photographic. Fifty wave-lengths between 3700 and $6800 \AA$. Measurements only for points near the $\operatorname{limb}(\sin \theta=0.98$ to $\sin \theta=\mathrm{r})$.

Osawa at the total eclipse of 9 May I948. Photoelectric measurements of the total intensity of the crescent.

The darkening curves of Peytureaux show a break at about 15,000 , which is more visible in the curves derived by Pierce. We have here an evidence of the absorption by the $\mathrm{H}^{-}$, but an additional absorber cannot be excluded.

Ramanathan's values are consistently higher than any other. Ballario and Godoli find a greater darkening for shorter wave-lengths up to the limb. The uncertainty of their results is $3 \%$ up to $\sin \theta=0.998$ and grows up to $20 \%$ for $\sin \theta=\mathbf{I}$, the agreement with ten Bruggencate's results (I949) being very good except for the points between $\sin \theta=0.998$ and $\sin \theta=\mathbf{I}$.

Osawa states that the solar crescent has a rapid decrease of brightness in the last $I \%$ of the radius.

The integral equation connecting the true and the apparent profile of the limb has been solved by Fellgett and Schmeidler by means of a Fourier transformation. This method allows a sharp control of errors. New methods of calculation for the reduction of limbdarkening observations have been introduced by Mineur and Peytureaux and by Sykes.

A boundary temperature of $4793^{\circ} \mathrm{K}$. has been deduced by Ramanathan from his own results, while Erika Böhm Vitense obtains $3800^{\circ} \mathrm{K}$. A revision of the U.V. and I.R. corrections leads to an effective solar temperature of $5780^{\circ}$.

In order to ascertain a possible difference of temperature between the poles and the equator of the Sun, Minnaert has compared the brightness of these two regions in red and ultra-violet. The comparison made with a photoelectric apparatus has given a negative result. 


\section{Wave-Length Variation and Displacement of the Fraunhofer Lines}

The Evershed effect has been carefully investigated by Kinman at Oxford. The displacements have been measured with a self-recording micrometer employing Evershed's positive-on-negative method. The maximum error in velocity is $0 . \mathrm{rm}$. $/ \mathrm{sec}$.

The motion of gases in a spot is found to consist of an outward horizontal radial velocity which increases from I km. $/ \mathrm{sec}$. at the edge of the umbra to $2 \mathrm{~km}$. $/ \mathrm{sec}$. in the middle zone of the penumbra and falls to zero well outside the spot. The tangential and vertical velocities are less than the errors of measurement. The maximum radial velocity increases with the umbral radius.

Employing the same method, Hart has found that the mean rotational velocity at the equator, after the elimination of the limb effect, is $I \cdot 9 r \pm 0.0 r \mathrm{~km}$. $/ \mathrm{sec}$, and that the effect of the scattered light on the measured velocity is negligible. Local variations of velocity, amounting to $\pm 0 \cdot 17 \mathrm{~km}$. $/ \mathrm{sec}$. have been found.

A centre-limb shift of $+0.020 \AA$ for the emission lines $\mathrm{H}_{3}$ and $\mathrm{K}_{3}$ has been found by Das and Rajeswara Rao at Kodaikanal.

\section{MEASUREMENTS OF WAVE-LENGTHS, IDENTIFICATIONS, Abundance of Elements and Compounds}

Interferometric measurements of solar wave-lengths have been performed at Oxford by Adam employing Treanor's method of 'circular channels'. A direct comparison with the primary standard has been made, obtaining wave-lengths of solar lines with a standard deviation of $\pm 0.0006 \AA$. The 'red shift' of solar lines, amounting to $0.0093 \AA$ at $5080 \AA$ and to $0.0065 \AA$ at $6020 \AA$, cannot be explained as a combination of relativity shift and a reasonable collisional shift.

The solar lines 3692 and $4368 \AA$ have been attributed to $\mathrm{O}_{\mathrm{I}}$ by Nikitin, who thinks, besides, that the infra-red line Io,I23 $\AA$ may be identified as a transition of He Ir. The same line has been attributed to the transition $3 p^{1} P_{1}-3 d^{1} P_{1}$ of $\mathrm{C}_{\mathrm{I}}$ by Edlén. Furthermore, Edlén has identified twenty faint solar lines as due to $\mathrm{C}_{\mathrm{I}}$, in addition to those already known, and eight infra-red lines as transitions of $\mathrm{Ca}$ II which have been observed in the hollow cathode discharge.

In the spectrum of the solar limb, Goldberg and Müller have found nearly 300 lines of $\mathrm{CO}$ belonging to six different bands; the abundance of solar CO computed is $\mathrm{I} \cdot 49 \times 10^{10} \mathrm{~mole}-$ cules per square centimetre above the photosphere. The excitation temperature derived is $4300^{\circ} \mathrm{K}$., suggesting a possible concentration of $\mathrm{CO}$ near the top of the photosphere.

Wave-length measurements of 430 lines, belonging to the $(2,0)$ band of the CN-red system, have been performed by Righini and Rigutti on a spectrum of the carbon flame. More than eighty new CN lines have been found in the solar spectrum in addition to the seventy already identified by Babcock and Moore.

The abundance of Tc has been investigated by Hubenet, de Jager and Zwaan. Two lines belonging to $T c$ II coincide with faint solar lines, but the resulting abundance of Tc is too great. The identification seems therefore doubtful.

A new determination of the abundance of deuterium has been made by de Jager obtaining $\mathrm{I} \cdot 45 \times \mathrm{IO}^{-4}$ with respect to hydrogen. Shklovsky obtains the abundance ratio $\mathrm{D} / \mathrm{H}$ of $\mathrm{ro}^{-6}$ from analysis of the flash spectrum, and Severny, from accurate profiles of $\mathrm{D} \alpha$ and $\mathrm{D} \beta$, derives a value of several times the Earth's abundance.

Gauzit identifies two faint infra-red lines as due to forbidden transitions of $\mathrm{NI}$, obtaining an abundance almost equal to that of $\mathrm{O}_{\mathrm{I}}$.

The abundance of $\mathrm{Be}$ in the Sun is the same as on the Earth according to Greenstein and Tandberg. This fact may indicate that Be in the Sun has never been at a sufficiently high temperature to be destroyed by thermonuclear reactions. Shklovsky thinks, instead, that $\mathrm{Li}$ and Be can be formed in the Sun as a result of a nuclear splitting by primary cosmic rays.

The presence of forbidden transitions belonging to NiII and $\mathrm{Fe}$ I has been studied by Swensson without reaching a conclusive identification. 


\section{Intensity and Profiles of Fraunhofer Lines}

The centre-limb variation of line profiles has been investigated by Priester at Göttingen with a Lummer plate giving a resolving power of 370,000. The temperature variations of the surface layers obtained by studying the $\mathrm{Na}-\mathrm{D}$ lines accords well with the solar model Vitense II.

A Fabry-Perot interferometer, with highly efficient multicoated layer, combined with a plane grating spectrograph (resolving power I,000,000), has been employed by von Klüber and Suemoto at Cambridge for the investigation of centre-limb variation of faint Fraunhofer lines.

Profiles of $\mathrm{H} \alpha$ and $\mathrm{H} \beta$ have been obtained by Severny with a photo-electric photometer. The blue wing of both lines shows a depression of $2 \%$, which is attributed to the presence of $\mathrm{D} \alpha$ and $\mathrm{D} \beta$. The profile of $\mathrm{H} \alpha$ has been observed by Pierce at McMath, employing a photo-electric photometer and a spectro-graph with a resolving power of I50,000. Both wings show a depression of $\mathrm{I} \%$ at $40 \AA$ from the centre.

Sherbina-Samoilova has investigated the profiles of sodium lines and their centre-limb variation.

The hyperfine structure of some metallic lines has been studied by Abt, showing how the structure contributes to the line-widening. Also, the asymmetry of the hyperfine structure pattern may give a shift in wave-length of about $0 \cdot 0$ I $\AA$.

A broad programme of research on the centre-limb variation of profiles is now in progress at the Göttingen Observatory. A high-resolution Lummer plate is employed for studying the profiles of two strong iron multiplets. The comparison with solar models is performed with the help of an electronic computer.

Temperature inhomogeneity due to granulation, deviations from thermodynamical equilibrium and effects of micro- and macro-turbulence will be taken into account. The contribution of each layer to the line-formation is calculated in order to obtain more information on the physical conditions of the higher part of the solar atmosphere.

A similar problem, concerning weak solar lines belonging to several elements, is now studied by Elste in Göttingen. Differences between observations and calculation seem to be due to a micro-turbulence of deep layers.

Equivalent widths and profiles of lines between $345^{\circ}$ and $375^{\circ}$ have been measured by Thompson at Dunsink Observatory. Photo-electric tracing of the whole solar spectrum is carried on by Osawa at Tokyo.

A new and very useful tabulation of Voigt functions, to be used for representing observed profiles, has been prepared by Elste.

The profiles of the hydrogen lines, belonging to the Balmer, Paschen and Brackett series, have been quantitatively explained by de Jager considering the solar atmosphere as a juxtaposition of elements whose temperature differs by $\pm 1000^{\circ} \mathrm{K}$. A temperature fluctuation of the same order has been assumed by Böhm in order to explain the centrelimb variation of line-profiles.

From a study of several hundred weak lines, measured in the Utrecht Photometric Atlas, Miss Bell deduced the following facts:

(a) for lines of different atomic weight (C, O, Si, Fe) but of fairly similar excitation potential the Doppler half-widths are inversely proportional to the square root of the atomic weight;

(b) for weak lines of different excitation potential but of the same atom ( $\mathrm{Fe})$, the Doppler half-width decreases with increasing excitation potential;

(c) for $\mathrm{Fe}$ I the damping half-width increases only slightly with excitation potential, but depends strongly on the parity of the lower term. This parity effect in Fe I (noticed by Carter in his curves of growth) appears to be adequately explained by the theory of van der Waals broadening by collisions with hydrogen atoms.

Miss Bell originally suggested that facts $(a)$ and $(b)$ indicated an absence of turbulence in excess of $0.5 \mathrm{~km}$. $/ \mathrm{sec}$. and a kinetic temperature ranging from $I I, 500^{\circ}$ at the base to $20,000^{\circ}$ at the top of the reversing layer. 
Houtgast showed that this conclusion is not valid, since the lines of differing atomic weight originate at very different depths and are therefore affected by very different damping. He finds for $\mathrm{C}, \mathrm{O}, \mathrm{Fe}$ an agreement between the profiles calculated for a model atmosphere of Claas and de Jager and those observed.

Measurements of polarization in the profile of $\mathrm{CaI} 4227, \mathrm{NaD}_{1}$ and $\mathrm{D}_{2}, \mathrm{Mgb}$ have been performed by Jäger at Göttingen. He obtains a polarization of $5 \%$ at the northern limb and one of $I \cdot 4 \%$ at the western limb. The results are in accordance with Thiessen's theory.

The prediction made by Hubenet that the equivalent width remains unchanged when longitudinal Zeeman effect is present, has been confirmed by Zwaan's measurements on the spectrum of a big spot where no 'magnetic intensification' has been detected.

An interpretation of profiles of strong Fraunhofer lines has been given by Miyamoto. The centre intensity is explained in terms of fluorescence, the line-width is attributed to turbulent motions whose velocity increases in the upper layers. A prediction of the Lyman alpha profile has been given by Miyamoto and by Jeffreys, but the agreement with the observation is very poor.

Interlocked multiplets have been investigated theoretically by Busbridge and Stibbs. A solar model from the equivalent widths has been obtained by Pecker and van Regemorter. A surface temperature of $4 \mathrm{I} 5 \mathrm{I} \pm 30$ has been deduced by van Regemorter from the equivalent width of titanium lines.

\section{CuRves of Growth}

Mitropanova has determined a new curve of growth for Fe lines employing $g f$ values measured by herself. An excitation temperature of $4535 \pm$ I Io has been obtained.

Lines widened by a hyperfine structure fit a particular curve of growth as shown by Abt; the parameters deduced in this case have not the usual meaning, since the lines consist of many closely spaced unresolved lines.

Exact curves of growth have been calculated by Wrubel for a Schuster-Schwarzschild model atmosphere.

The equivalent widths of many weak lines belonging to vanadium and titanium have been measured by Bretz at Göttingen, applying Jäger's method of the 'effective linewidth'. The purpose of this study is to investigate the centre-limb variation of the curve of growth.

\section{Far Ultra-violet Spectrum Survey}

Rocket spectra of the ultra-violet part of the solar spectrum have been analysed by Clearman and by Wilson, Tousey, Purcell, Johnson and Moore. Lines of As I and Ru II have been found; other lines of $\mathrm{Pt} I$ and $\mathrm{Be} I$ are still uncertain. Atomic lines of $\mathrm{Be} I$ have been identified and no solar or terrestrial bands are present in these spectra.

A relative abundance of $\mathrm{Mg}$ to $\mathrm{Ca}$ has been deduced from the emission lines of $\mathrm{Mg}$ II and from $\mathrm{H}$ and $\mathrm{K}$ of $\mathrm{Ca}$ II.

The limit of the ultra-violet spectrum has been shifted to $7 \AA$ according to Byral, Chubb and Friedman, who observed X-rays by means of a photon counter carried by a rocket to a height of more than $87 \mathrm{~km}$. The intensity of X-rays, with $\lambda>$ Io $A$, grows steeply within the ionospheric E-layer.

With the same technique, the radiation of $\mathrm{L} \alpha$ has been detected at an altitude greater than $74 \mathrm{~km}$. Later on, Pietenpol and others succeeded in photographing $\mathrm{L} \alpha$ which has a width of about $6 \AA$.

Continuous recording of the ultra-violet and X-ray spectrum of the Sun has been planned by Singer with photo-counters carried by an artificial satellite circulating in an orbit at a height of $400 \mathrm{~km}$. 


\section{INFRA-RED SPECTRUM SURVEYS}

A new infra-red atlas of the solar spectrum between 2.80 and $23.73 \mu$ is now being prepared at Liège by Migeotte and Swensson with the collaboration of Neven. It will contain I22 plates giving reproductions of the solar spectrum recorded with high dispersion at the Jungfraujoch Scientific Station (Switzerland). Owing to the low temperature and the high altitude of the station the perturbing action of the $\mathrm{H}_{2} \mathrm{O}$ bands is greatly reduced.

A table containing wave-lengths and equivalent widths of 888 infra-red lines between I.4 and 2.5 $\mu$ has been published by Mohler, Pierce, McMath and Goldberg. The wavelength error is on the average $\pm 0 \cdot 15 \AA$. More than 400 lines remain unidentified.

Shaw, Oxholm and Claassen have recorded the solar spectrum between 7 and I3 $\mu$, finding 800 lines produced chiefly by atmospheric water vapour. Bands of $\mathrm{CO}_{2}, \mathrm{CH}_{4}$, $\mathrm{N}_{2} \mathrm{O}$ and $\mathrm{O}_{3}$ have been found, but many lines remain still unidentified.

High-dispersion solar spectra between 2 and $5 \mu$ have been obtained at the Dominion Observatory (Canada).

Identifications of many infra-red multiplets belonging to several elements have been made by $\mathrm{Ch}$. Moore.

\section{TELIURIC Lines and Bands}

The $\mathrm{CO}$ content of the atmosphere has been investigated by Benesch, Migeotte and Neven at the Jungfraujoch, finding daily fluctuations which are independent of the meteorological situation. No evidence of a geographical distribution of $\mathrm{CO}$ on the earth has been found. The results of Goldberg and McMath on the abundance of $\mathrm{CH}_{4}$ and $\mathrm{N}_{2} \mathrm{O}$ and their exponential decrease with height have been confirmed. No conclusive evidence of the existence of NO has been found.

The abundance of $\mathrm{CO}$ over Ottawa has been determined by Locke and Herzberg, who also found that the intensity ratio between the fundamental and the overtone bands of $\mathrm{CO}$ agrees with the value measured in the laboratory by Penner and Weber.

New lines belonging to the molecules ${ }^{12} \mathrm{C}{ }^{16} \mathrm{O}_{2}, \mathrm{~N}_{2} \mathrm{O}$ and $\mathrm{O}_{3}$ have been identified by Migeotte, Neven and Swensson.

Shaw and Howard have identified a new $\mathrm{O}_{3}$ band at about $4 \cdot 8 \mu$.

Results on the distribution of ozone in our atmosphere are reported in the volume Rocket Exploration of the High Atmosphere. Johnson, Purcell and Tousey give the results obtained by measuring the absorption of the solar ultra-violet radiation, to a height of $70 \mathrm{~km}$., with photon counters carried by a rocket; Regener, Petzold and Ehmert report the results obtained with spectrographic equipment carried by balloons and the results deduced from lunar eclipse observations.

Measurements of the zenith brightness, up to $r 35 \mathrm{~km}$., have shown the existence of a 'Day airglow' which has an intensity ${ }^{4} 0^{4}$ times the night airglow. Its intensity is still $3 \%$ of the day-time ground value.

\section{GRANULATION}

The diameter and lifetime of granules have been investigated by Macris on film taken at the Pic du Midi by Lyot. It appears that the diameters follow a Gaussian distribution and are slightly lengthened in the E.-W. direction. The mean diameter is $I " 5$ and the lifetime ranges from 2 to 9 minutes.

Visual measurements performed by Thiessen with a $60 \mathrm{~cm}$. refractor at Hamburg support Macris' results concerning the diameters; he finds that the granules have a polygonal shape which disappears if the resolving power is not high enough.

A new analysis of Doppler shifts due to turbulent motions of granules has been performed by Frenkiel and Schwarzschild. They find that the turbulence spectrum has a primary maximum at $300 \mathrm{~km}$. and a secondary one at $15,000 \mathrm{~km}$. Correlation analysis of turbulent velocities and brightness of photospheric granulation, performed by Stuart 
and Rush, suggests a regular granular structure with a dimension of the order of $2300 \mathrm{~km}$. Furthermore, auto-correlation of brightness fluctuations, derived from plates obtained by Miller, reveal a maximum at about $14,000 \mathrm{~km}$. which is related to the finding of Frenkiel and Schwarzschild. According to Schwarzschild the bulk of the energy in the photospheric turbulence occurs in elements too small to be resolved.

Thiessen does not agree with this point of view, since the difference between the mean turbulent velocity, $\pm 0.37 \mathrm{~km}$. $/ \mathrm{sec}$., deduced by Schwarzschild and Richardson from the Doppler effect of granules, and the velocity of $\pm I \cdot 7 \mathrm{~km}$. $/ \mathrm{sec}$., deduced from line profiles and curves of growth, may. be explained as an effect due to scattered light. The difference of brightness between granules and intergranular spaces, as observed by Thiessen, amounts to $35 \%$ at $5650 \AA$; the one observed by Schwarzschild and Richardson is, on the contrary, only $6 \%$. Since correction for scattered light leads to a line shift, the mean turbulent velocity becomes $\pm \mathrm{I} \cdot 85 \mathrm{~km}$. $/ \mathrm{sec}$., instead of $\pm 0.37 \mathrm{~km}$. $/ \mathrm{sec}$.

A model for the convective hydrogen zone has been suggested by Vitense. A new theory of the granulation has been developed by Schatzman.

\section{SPECTRA OF SUNSPOTS}

Spectrophotometric measurements have been performed by Michard at Meudon, by Das and Ramanathan and by Ananthakrishnan at Kodaikanal. The intensity ratio spot to photosphere is found to decrease with decreasing wave-length, to be almost independent of the position of the spot and to decrease with the increasing spot size. Michard also finds that the geometrical depth is greater and the pressure smaller in the spot than in the photosphere.

Gurtovenko has investigated the wave-length shifts of the lines $\mathrm{K}_{2}$ and $\mathrm{K}_{3}$ in the spots. Zhuralev finds that the spectral type of an average spot is G8.

Lines belonging to the $(2,0)$ band of $C O$ have been found seven times enhanced in the spots by Locke and Herzberg.

\section{Chromosphere and Prominences}

The abundance of $\mathrm{Mg}, \mathrm{Al}, \mathrm{Ca}$ and $\mathrm{Na}$ seems to be greater in the chromosphere than in the photosphere, as has been pointed out by Schwartz. The temperature of the low chromosphere has been obtained by Goldberg from the Lyman alpha profile. The value of $6000^{\circ} \mathrm{K}$. deduced supports the Woolley-Allen chromospheric model. The same conclusion is obtained by Miyamoto.

Goldberg, Dodson and Müller find that the width of $\mathrm{H} \alpha$ in solar flares increases steadily with increasing distance of the flare from the central meridian. The same effect has been found by Righini on normal flocculi.

The fact that the $\mathrm{He} \mathrm{I}$ line at $\lambda$ I0,830 appears in the active regions of the solar disk is interpreted by Nikitin as a consequence of an accumulation of atoms in the $2^{3} S$ metastable state.

A new theory of spectroheliograms has been advanced by Reichel.

Thickness of prominences has been deduced by Haug and by Koelbloed applying Zanstra's theory to the intensity of the continuum measured on eclipse spectra. The values derived are higher than Pettit's estimates. Redman and Zanstra proved in the same way that the thickness of the prominence, observed at the I952 eclipse, may be 40,000 or $6000 \mathrm{~km}$., according to the density and the electron temperature adopted. As this last value agrees with measurements performed on spectroheliograms, the proton density $5 \times \mathrm{IO}^{10} \mathrm{~cm} \cdot .^{-3}$ and the electronic temperature $15,000^{\circ} \mathrm{K}$. seem to be the most likely results.

Measurements of equivalent widths and profiles with high dispersion performed by ten Bruggencate on $\mathrm{H}$ and $\mathrm{He}$ lines, give an excitation temperature of $3700^{\circ} \mathrm{K}$., a kinetic temperature of $13,000^{\circ} \mathrm{K}$. and no turbulence.

The spectrum of a prominence has been observed by Righini at the eclipse of I952. Among the forty-eight lines observed, the infra-red $\mathrm{O}_{\mathrm{I}}$ triplet has been identified. 


\section{The Corona}

Polarization of the $\mathrm{F}$ corona due to an orientation of grains under the action of a weak magnetic field has been predicted by Thiessen. The identification of the yellow coronal line has been confirmed by Pecker, Billings and Roberts. From the profiles one may conclude that the element which produces the line must have an atomic weight of 40 in agreement with Edlén's identification of Ca XV.

The red coronal line appears widened on active places, indicating a higher temperature or a strong local turbulence. This result has been obtained by Billings, Pecker and Roberts, comparing the profiles of this line on several places of the corona.

Measurements of equivalent width of the green coronal line, referred to the spectrum of the centre of the disk, have been performed by Kraul in order to obtain an absolute calibration of the intensity scale employed at the Wendelstein Observatory.

Interferometric measurements of the width of 5303 have been obtained by von Klüber and Jarrett at the eclipse of I954 employing a multicoated Fabry-Perot interferometer. A substantially improved interferometer has been prepared for the eclipse of June r955.

The new coronal lines $\lambda 3997,435 \mathrm{I}, 44 \mathrm{I} 2$ have been found by Dollfus on spectra taken by Lyot at Khartoum. Identifications of four coronal lines have been given by Edlén as follows: 4412.4, A XIV; 435I, Co Xv; 3997, Cr XI; 30Io, Fe XII.

The spectral curve of the corona in the range of soft X-rays has been calculated for several temperatures by Elwert. It appears that between 30 and $5^{\circ} \AA$ the emission has a maximum. This radiation in the high atmosphere of the Earth gives an electronic density which is in accordance with the one observed in the E-layer.

\section{MAGNetic Freld}

A report on the general magnetic fields in the Sun and the stars has been published by Babcock and Cowling (M.N. I13, 357, 1953). Another report on 'Spectroscopic measurements of magnetic fields on the Sun' has been written by von Klüber for Vistas in Astronomy (Pergamon Press, London, r955).

The new solar magnetograph of $\mathbf{H}$. W. Babcock was put into regular operation at the beginning of 1953 at the Hale Solar Laboratory in Pasadena. The instrument can record weak magnetic fields down to I gauss, the noise level being only $0 \cdot I$ gauss. The results are displayed on a cathode-ray oscillograph.

According to Billings it seems that the solar regions having magnetic activity show an optical activity (spots, flares, etc.) about one rotation later.

The old measurements by Hale of the general magnetic field have been newly discussed by von Klüber in order to see how the Zeeman pattern of the lines may have influenced the final results. An attempt to detect a general magnetic field of the Sun by combining a Lummer plate with a plane grating spectrograph has been made by von Klüber at Cambridge. Although the resolving power was about 600,000 , no systematic field of more than 2 gauss could be found.

The perturbing action of turbulent motions in the solar atmosphere on the measurements of weak magnetic fields has been pointed out by Alfvén and by Kiepenheuer. According to these authors it is not possible at present to draw any conclusion about the value of the general magnetic field of the Sun from measurements of the Zeeman effect.

If the axis of symmetry of the coronal polar rays represents the magnetic polar axis, the magnetic poles are not antipodal and they do not coincide with the rotation poles. This conclusion is reached by Abetti from the study of many good photographs of the corona.

The measurements of the magnetic field of spots performed at Potsdam have been employed by Schröter for tracing the distribution of the magnetic field on the surface of a spot. The radial distribution of the field, according to Mattig, does not agree with Broxon's formula and a modified formula has to be used.

Discussing this matter, Thiessen remarks that, owing to the partial overlapping of the 
Zeeman components, the measured fields are smaller than the real ones and that measurements ought to be corrected in consequence of this effect. Then Broxon's formula satisfactorily represents the measured field distribution.

The magnetic polarity and the maximum field intensity of all spots observed in Potsdam over the period I946-5I have been published by Grotrian.

Some investigation of spot magnetic fields has been made by von Klüber at Cambridge. The infra-red Si I line at ro,289 $\AA$, having a very large simple Zeeman splitting, has been found very convenient for such investigations.

\section{INSTRUMENTS}

Description of the solar installation of the Dunsink Observatory and its performance has been published by Brück. The new high-resolution spectrograph at the Sacramento Peak Observatory is nearly completed. It will be employed to obtain solar and sunspot spectra for special studies.

Suemoto, Hokugo and Shimizu report that a plane grating spectrograph of $5 \mathrm{~m}$. focal length has been installed at the Tokyo Observatory. The spectrum from 3600 to $6600 \AA$ may be taken with a single exposure on commercial $35 \mathrm{~mm}$. film, making it possible to study the line-profiles and intensity of prominences and flares.

A concave grating spectrograph on a Wadsworth mounting has been put into operation at the Georgetown College Observatory as reported by Kiess and Heyden. The spectrograph has a focal length of $7 \mathrm{~m}$. and is equipped with two concave gratings covering a spectral range from 3000 to $13,000 \AA$.

At Cambridge (England), the large solar spectrograph $(f=9 \mathrm{~m}$.) has been equipped with a $20 \times 14 \mathrm{~cm}$. grating, blazed in the $5^{\text {th }}$ order, giving a practical resolving power of 600,000 . A spectroscopic laboratory with all necessary equipment has also been organized.

Combining this spectrograph with a multicoated Fabry-Perot interferometer, von Klüber has easily obtained a resolving power of $1,000,000$. In collaboration with Beggs, von Klüber is preparing equipment using photomultipliers, polarizing optics and electrooptical retardation plates, for recording weak magnetic fields, as well as small line-shifts with this instrument.

An improvement on auto-collimating spectrographs has been introduced by Minnaert. He showed that the astigmatism may be entirely avoided by inclining the lens so that the normal moves perpendicularly to the plane of incidence. At the same time reflected images are avoided.

A photoelectric photometer with two photomultipliers, for measuring the intensity distribution in the solar spectrum, has been mounted on the $7 \mathrm{~m}$. concave grating spectrograph of the Dunsink Observatory.

Kuprevich reports on the very rapid photometer, set up at the Pulkovo Observatory, employed with a plane grating spectrograph. This instrument will be used for obtaining accurate profiles and Fraunhofer line intensities.

As a standard source for absolute radiation measurements, a black body model has been constructed by Sitnik at Moscow.

\section{TABLES OF Fraunhofer Lines}

This co-operative enterprise was started by Mrs Moore-Sitterly, Dr H. C. Babcock and the Utrecht Observatory, the last contributing with the equivalent widths measured from the Utrecht Photometric Atlas. As of I January I955, the equivalent widths of the lines had been measured between 8700 and $4000 \AA$. 


\section{BIBLIOGRAPHY}

\section{(I) Energy Waye-length Curve and Solar Constant}

F. S. Johnson, J. D. Purcell et al.: Rocket Exploration of the Upper Atmosphere. Pergamon Press, London, 1954, p. 279.

R. Stair, R. G. Johnston and T. C. Blagg: J. Res. Nat. Bur. Standards, 53, II3, I954.

A. K. Pierce: (see section (2)).

R. Peytureaux: (see section (2)).

L. B. Aldrich and W. H. Hoover: Ann. Astroph. Obs. Smithsonian Inst. 7, 1954.

G. C. Abbot: Smithsonian Inst., Misc. Coll. 122, no. 4.

W. B. Rimmer and C. W. Allen: Mem. Commonwealth Observatory, no. II, 1950.

\section{(2) Centre-limb Darkening}

R. Peytureaux: Ann. d'Astrophys. 15, 302, 1952; C.R. 236, 896, I953; C.R. 238, 867, I954.

A. S. Ramanathan: Ann. d'Astrophys. 17, 18, 1954.

A. K. Pierce: $A p . J$. 120, 22 I, 1954.

P. ten Bruggencate, H. Gollnow and F. Jäger: Zs. Ap. 27, 223, 1950.

G. Righini, C. Ballario and G. Godoli: Mem. Società Astr. Ital. xxıv, 3, 1953.

C. Ballario and G. Godoli: Mem. Soc. Astr. It. XxvI, 73, I955.

K. Osawa: Ann. Tokyo Astr. Obs. II series, 3, 53, 1951.

P. Fellgett and F. Schmeidler: M.N. x12, 445, 1952.

J. B. Sykes: $M . N$. 113, 198, 1953.

E. Böhm Vitense: $Z s . A p .34$, 209, I954.

M. Minnaert: Acc. Nazionale dei Lincei, XI Convegno Volta, p. 50, Roma, I953.

\section{(3) Wave-rength Variation and Displacement of the Fraunhofer Lines}

H. H. Plaskett: $M . N$. I12, II7, I952.

T. D. Kinman: M.N. 112, 129, 1952.

T. D. Kinman: M.N. 1r3, 237, 1953.

A. B. Hart: $M \cdot N$. 114, 275, I954.

A. K. Das and Rajeswara Rao: Kodaikanal Bulletin, no. I24.

(4) Measurements of Wave-length, Identifications, Abundafce of Elements AND COMPOUNDS

M. G. Adam: M.N. 112, 129, I952.

A. Nikitin: Ann. Leningrad Univ. no. I53, 1952.

B. Edlén: Acc. Nazionale dei Lincei, XI Convegno Volta, p. 56, Roma, 1953.

L. Goldberg and E. A. Müller: $A p . J$. 118, 397, 1953.

G. Righini and M. Rigutti: Mem. Soc. Astr. It. Xxv, 3 II, 1954. Osservazioni e Memorie del-

l'Osservatorio di Arcetri, fasc. 69, 1954.

H. Hubenet, C. de Jager and C. Zwaan: Les Processus Nucléaires dans les Astres, P. 47I, Liège, I954.

I. S. Shklovsky: A.J. U.S.S.R. (in the Press):

A. B. Severny: (see section (5)).

J. Gauzit: Les Processus Nucléaires. ., p. 468, 1954.

J. Greenstein and E. Tandberg: $A p$. J. I19, I13, I954.

J. Swensson: Bull. Acad. Roy. de Belgique, 38, 705, 1952.

\section{(5) Intensity and Profiles of Fraunhofer Lines}

W. Priester: $Z s . A p \cdot$ 32, 200, I953.

A. B. Severny: Comptes Rendus de l'Acad. d. Sci. U.R.S.S. 97, no. 5, 1954. A.C. U.S.S.R., no. I 50,1954 .

$$
\text { I76 }
$$


A. K. Pierce: $A p . J$. 120, 233, I954.

I. S. Scherbina-Samoilova: A.J. U.S.S.R. 30, 4 $4^{\mathrm{I}}$, I953.

A. Abt: $A p . J$. 115, 199, 1952.

G. Elste: $Z s . A p .33,39$, I953.

C. de Jager: Nature, Lond., 173, 680, 1954 .

K. H. Böhm: Zs. Ap. 35, 179, I954.

B. Bell: $A$ study of Doppler and damping effect, etc. Thesis, Harvard University, r95 I.

J. Houtgast: Acc. Nazionale dei Lincei, XI Convegno Volta, p. 33, Roma, 1953.

F. W. Jäger: Zs. Ap. 34, 237, 1954.

H. Hubenet: $Z s$. $A P$. 34, I Io, 1954.

S. Miyamoto: $Z s . A p$. 3I, 282, I953; Zs. Ap. 35, I45, 1954.

S. Miyamoto: Publ. University Kyoto, no. $3^{6}$.

J. T. Jefferies: Austr. J. Phys. 6, 22, 1953.

I. W. Busbridge and D. W. N. Stibbs: M.N. r14, 2, 1954 .

J. C. Pecker and H. van Regemorter: C.R. 234, I850, I953.

(6) Curves of Growth

L. A. Mitropanova: Izvestia Glav. Astr. Obs. Pulkovo, xIx, no. 149, 1952.

A. Abt: $A p . J$. 115, 199, I952.

M. H. Wrubel: $A p . J$. I19, 5 I, I954.

(7) Far Ultra-violet Spectrum Survey

H. E. Clearman: $A p . J .117,29,1955$.

N. L. Wilson, R. Tousey, J. D. Purcell and F. S. Johnson: $A p . J$. Ir9, 590, 1954.

E. T. Byram, T. Chubb and H. Friedman: Rocket Exploration of the Upper Atmosphere. Pergamon Press, London, 1954, pp. 274-6.

S. F. Singer: Rocket Exploration of the Upper Atmosphere. Pergamon Press, London, I954, p. 368 .

(8) INfra-Red Spectrum Surveys

M. Migeotte and A. H. Nielsen: Bull. de la Classe de Science de l'Acad. Royale de Belgique, 38, 5, 1952 .

O. C. Mohler, A. K. Pierce and R. R. McMath, L. Goldberg: Ap. J. II7, 4I, 1953.

J. H. Shaw, M. L. Oxholm and H. H. Claassen: Ap. J. 116, 554, 1952.

C. E. Moore: J.O.S.A. 43, Ior4, I953.

(9) Telluric Lines and Bands

W. Benesch, M. Migeotte, L. Neven: J.O.S.A. 43, I I19, 1953.

M. Migeotte and L. Neven: Mém. Soc. Roy, des sc. de Liege, 12, I65, 1952.

J. L. Locke and L. Herzberg: Can. J. Physics, 3I, 504, 1953.

M. Migeotte, L. Neven and J. Swensson: $N$. Cimento, serie IX, suppl. al volume XII, 1954.

J. H. Shaw, J. N. Howard: $A p . J$. II7, 460, I953.

\section{(io) Granulation}

C. Macris: Ann. d'Astrophysique, 16, I9, I953.

G. Thiessen: Zs. Ap. 35, 237, 1955.

F. N. Frenkiel and M. Schwarzschild: $A p . J .116,422,1952$.

F. E. Stuart and J. H. Rush: $A . J .58,231$, 1953.

E. Vitense: $Z s . A P .32$, I35, 1953.

E. Schatzman: Acad. Roy. des Sciences Belgique; Bull. Cl. Sciences, 39 and 40, I953. 
(i I) Spectra of Sunspots

R. Michard: Ann. d'Astrophysique, r6, 2 I 7, 1953.

A. K. Das and A. S. Ramanathan: Zs. Ap. 32, 91, 1953.

A. S. Ramanathan: Zs. Ap. 34, 169, I954.

R. Ananthakrishnan: Proc. Indian Acad. Science, 37, 1953.

Gurtovenko: Astr. Circ. U.S.S.R. no. I I3, 1953.

S. S. Zhuralev: Astr. Circ. U.S.S.R. no. I26, 1952.

(i2) Chromosphere and Prominences

S. Schwarz: $A p . J .119,296,1954$.

L. Goldberg: $A p . J$. 120, 185, 1954 .

L. Goldberg, H. Dodson, E. A. Müller: $A p . J$. 120, 83, 1955.

A. A. Nikitin: $A . J . U . S . S . R .29,463,1952$.

M. Reichel: Zs. $A p$. 33, 79, 1953 .

S. G. M. Haug: Circ. Astr. Inst. Amsterdam, no. 5, 1952.

R. O. Redman and H. Zanstra: Circ. Astr. Inst. Amsterdam, no. 6, 1952.

P. ten Bruggencate: Atti XI Convegno Volta, 163, 1953; Veröff. Gött. 104.

G. Righini: Atti XI Convegno Volta, 166, I953.

(13) The Corona

G. Thiessen: Zs. Ap. 32, I 73, I953.

Ch. Pecker, D. E. Billings and W. O. Roberts: $A p . J$. 120, 209, I954.

Ch. Pecker, D. E. Billings and W. O. Roberts: C.R. 238, I I94, I954.

W. Kraul: Zs. Ap. 33, I 74, 1953.

A. $H$. Jarrett and $H$. von Klüber (in the Press).

A. Dollfus: C.R. 236, 996, 1953.

G. Elwert: Mitt. Astr. Inst. Univ. Tübingen, no. $1 ; 8 ; 9$.

(i4) Magnetic Field

H. W. Babcock: Ap. J. 118, 387, 1953.

D. E. Billings: $A p . J . \mathbf{2 0}, 184,1954$.

H. von Klüber: $M . N$. II2, 183, I952.

K. O. Kiepenheuer: Mitt. Fraunh. Inst. no. Io.

G. Abetti: Atti XI Convegno Volta, p. 269, 1953.

E. H. Schröter: $Z s . A p \cdot 33,20,1953$.

W. Mattig: Zs. Ap. 31, 273, 1953.

G. Thiessen: Naturwissenschaften, 40, 2 I8, 1953.

W. Grotrian: Publ. Potsdam B 29, Heft 4, 1953.

H. von Klüber: Camb. Univ. Obs. Report, I951.

(15) INSTRUMENTS

C. C. Kiess and F. J. Heyden: J.O.S.A. 42, 876, 1952.

N. F. Kuprevich: Izvestia Glav. Astr. Obs. Poulkovo, xIX, I40, 1952; A.J. U.S.S.R. 31, 85. 1954.

Report of meeting. I September I955

President: Prof. G. Righini.

Secretary: Miss Dr. E. A. Müller.

All members of Commission I2 request the inclusion of the bibliography in the Draft

Report since the Transactions are likely to be used as a reference and working book.

Prof. Minnaert then gives a short description of the progress in preparing the solar

I78 
spectroscopic tables for publication. They will soon be completed and will contain the spectroscopic data and the equivalent widths of all solar lines between the near ultraviolet (from records of the Dunsink Observatory) and the far infra-red (from Mohler's tables and Migeotte's atlas). The far ultra-violet spectra obtained with rockets will not be included. The request for a grant of 2000 dollars, in addition to the I 660 dollars already allotted at the Rome meeting for the publication of these tables, is approved by the commission (Resolution A).

In view of the very great importance of atomic constants in astrophysical research, Prof. ten Bruggencate suggests that all astrophysicists should get in touch with their colleagues in the physics department of their universities and stress the need for experimental determinations of $f$-values, cross-sections, etc. Mrs Moore Sitterly, being a member of the Joint Commission on Spectroscopy which deals with some of these problems, agrees to recommend the resolution of Commission I2 (Resolution B) at the meeting of that commission.

Dr Tousey then reports on work on 'Emission Lines in the Extreme Ultra-violet Spectrum of the Sun' being done by F. S. Johnson, H. H. Malitson, J. D. Purcell and R. Tousey at the U.S. Naval Research Laboratory in Washington, D.C.

Culminating a long series of experiments, a spectrograph, flown in an Aerobee rocket to an altitude of II $5 \mathrm{~km}$. on 21 February 1955, was successful in producing a spectrum of the Sun that showed at least 30 emission lines in the wave-length range $1817-977 \AA$. The dispersion was $4^{\mathrm{I}} \AA / \mathrm{mm}$., produced by a 600 line $/ \mathrm{mm}$., $40 \mathrm{~cm}$. radius grating used near normal incidence in the first order. A 30 sec. exposure was made on Eastman SWR film. An astigmatic image of the Sun was formed on, and in front of, the slit with a toroidal mirror in such a way that the spectrum was approximately stigmatic in the extreme ultra-violet. Pointing of the spectrograph at the Sun during the flight with a maximum error of 2 minutes of arc was accomplished with a photo-electric servo-mechanism developed by the University of Colorado and known as the Biaxial Pointing Control.

The thirty emission lines definitely present have been identified. The Lyman alpha line of hydrogen was far stronger than any other line. Its intensity in erg $\mathrm{cm} .^{-2} \mathrm{sec} .^{-1}$ appeared to be $0 \cdot 4$, as against a value of $1 \cdot 0$ from our spectra on 2 February 1954 , and a value of $0 \cdot 1$ obtained by Byram et al. with photon counters on 5 May 1952. The Sun was believed to be quiet on all three days. The true width of the line cannot be determined since the spectrographic slit was equivalent in width to I $\AA$, but it was concluded that it was less than $0.3 \AA$, from a comparison with exposures made before flight with a laboratory source. Lyman beta was also recorded. Lyman gamma does not reach $I I 5 \mathrm{~km}$. because of strong attenuation by $\mathrm{N}_{2}$.

The resonance lines of $\mathrm{O}$ VI, at $103 \mathrm{I} \cdot 9$ and $1037.5 \AA$ were clearly present. Also recorded was the $\mathrm{N} \mathrm{v}$ resonance line at $1238.8 \AA$; the fainter component of this doublet was missing, presumably because of strong attenuation by atmospheric $\mathrm{O}_{2}$.

Almost all other expected strong lines requiring less excitation than $\mathrm{N} \mathrm{v}$ and occurring in the spectral range down to $977 \AA$ were recorded. These include the resonance lines of $C_{I}$, II, III, and IV, the resonance lines of Si II, III, and IV, the triplet of O I containing the ultimate line at $\mathrm{I} 302.2 \AA$, the ultimate line of S I, and the line of $\mathrm{He}$ II at $\mathrm{I} 640.5 \AA$, corresponding to $\mathrm{H} \alpha$. The ultimate line of Al II may be present, but is blended with an emission line of Fe II, a few weak emission lines of which seem to be present.

The solar continuum was observable to $I 55^{\circ} \AA$, where it disappeared in the stray-light background. Fraunhofer lines could be followed to $165^{\circ} \AA$. A sharp decrease in intensity of the continuum occurred on the short wave-length side of $2085 \AA$, with loss of strong Fraunhofer structure. Possible contributors to this solar absorption are the wings of Lyman alpha, continuous absorption by metallic atoms, such as $\mathrm{Ca}$ and $\mathrm{Al}$, and solar absorption by molecules such as $\mathrm{CO}$ and NO.

Since the spectrograph was in effect stigmatic, it was possible to examine the lines for limb brightening or darkening. The radiation was received from a narrow near-diametral strip of the Sun approximately parallel to its polar axis. In general the emission lines were quite uniform and exhibited neither limb darkening nor brightening. The $\mathrm{O}$ vi lines, however, were 
definitely brightened near the limb, compared to Lyman beta. The same was true of the He II and $\mathrm{C}$ IV lines relative to $\mathrm{C}$.

The ionization potential of $\mathrm{OV}$ and $\mathrm{N}$ rV are $113.3^{8}$ and $77.09 \mathrm{eV}$. respectively. Therefore, these radiations must originate in the top of the chromosphere or bottom of the corona where the temperature is sufficiently high, that is, of the order of $10^{5}$ degrees.

The full account of this work is expected to appear in 1956 as a paper by F. S. Johnson, H. H. Malitson, J. D. Purcell and R. Tousey in the Astrophysical Journal.

Dr Tousey remarks that the work is being continued and that his group hopes to fire rockets to altitudes higher than $200 \mathrm{~km}$. in order to avoid the strong oxygen absorption of the lower regions of the atmosphere. For the International Geophysical Year, the launching of rockets is being planned by the N.R.L. in close collaboration with the visual observations of solar flares reported at Boulder, Colorado. In the discussion that follows Dr Tousey's report, Drs Allen, Ellison, Goldberg, de Jager, Michard, Newkirk, Ǒhman and Saha take part.

The second paper read at this meeting is Dr Goldberg's report on some solar spectra taken with the new solar vacuum spectrograph of the McMath-Hulbert Observatory. This spectrograph is equipped with a Babcock grating blazed in the $5^{\text {th }}$ order, and is evacuated in order to avoid air turbulence within the long focal length spectrograph which would shift the lines by about 0.05 to $0.06 \AA$. The clear separation of the close iodine doublet at about $5400 \AA$ shows that the expected resolving power of about 600,000 is readily obtained. The peculiarity of all solar lines taken with this spectrograph is their irregular zig-zag shape, demonstrating the Doppler shift of the granules. The maximum displacement is about $\mathrm{I} \cdot 5$ to $\cdot 2 \cdot 0 \mathrm{~km}$. / $\mathrm{sec}$., the magnitude of displacement changing with the position on the disk, showing greater displacements near the solar limb. As the edge of the Sun is approached, the solar lines broaden. The fluctuations can also be observed in the continuum between the Fraunhofer lines, a dark horizontal line appearing in the continuum where the spectral lines have a 'moustache'. The atmospheric lines, on the contrary, appear completely straight. Thus it is easy to distinguish solar from telluric lines. Of particular importance is the shape of the $\mathrm{H} \alpha$ line the width of which fluctuates as much as $40 \%$, corresponding to local fluctuations of the chromospheric temperature of the order of a factor of 2 . Messrs Das, Plaskett and de Jager take part in the discussion that follows Dr Goldberg's report, and in this connexion Prof. ten Bruggencate communicates that at the solar tower at Göttingen they have started to study the stratification of the solar photosphere. With a Mount Wilson grating they observed in the $4^{\text {th }}$ order the infra-red oxygen triplet $(7772-7775 \AA)$ at the centre of the solar disk, and found a distinct asymmetry of the line profiles, the violet wing being stronger than the red wing. This can be explained with the three-stream model of Böhm, taking into account the temperature inhomogeneity and the Doppler displacement. The excitation potential of the oxygen lines is so high that the hot ascending elements of granula contribute much more to the line profile than the cooler descending elements. The asymmetry disappears if the lines are observed near the solar limb.

\section{Resolutions}

I. Commission I 2 strongly recommends that a sum of 2000 dollars be granted for the publication of the Solar Spectroscopic Tables, which are expected to be ready before the next meeting of the I.A.U. in 1958 . These tables are of very great importance for all solar and stellar spectroscopic work.

2. The Commission strongly recommends to all adequately equipped physical and astrophysical laboratories the urgently needed experimental determination of oscillator strengths ( $f$-values) and other astrophysical constants. 